\title{
Beta-arrestin2 deficiency protects against hepatic fibrosis in mice and prevents synthesis of extracellular matrix
}

\author{
Wu-Yi Sun ${ }^{1,2}$, Yuan-Jing Gu ${ }^{1,2}$, Xin-Ran $\mathrm{Li}^{1,2}$, Wen-Ting Peng ${ }^{1,2}$, Jing-Yu Chen ${ }^{1,2}$, Yang Ma ${ }^{1,2}$, Qing- \\ Tong Wang ${ }^{1,2}$, Wei Wei ${ }^{1,2}$
}

${ }^{1}$ Institute of Clinical Pharmacology, Anhui Medical University, China, ${ }^{2}$ Key Laboratory of Anti-inflammatory and Immune Medicine, Ministry of Education, China

Background: beta-arrestin2 is a multifunctional adaptor protein, which has recently been found to play new roles in regulating intracellular signalling networks associated with cell functions. Here we examine the role of beta-arrestin2 in the pathobiology of hepatic fibrosis. Methods: Clinical specimens from patients with hepatic fibrosis were used to observe the expression of beta-arrestin2 by immunohistochemistry. In vivo studies, liver fibrosis model was established in male Wistar rats. The dynamic expressions of beta-arrestin2, collagen I, collagen III, tissue inhibitor of metalloproteinase-1 (TIMP-1), matrix metalloproteinase-13 (MMP-13) in rat liver tissues were measured by immunohistochemical staining and Western blot. beta-arrestin2-/- mice were used to investigate the role of beta-arrestin2 in carbon tetrachloride (CCl4)-induced liver fibrogenesis. In vitro, hepatic stellate cells (HSCs) overexpression beta-arrestin2 were applied to explore the mechanism. Results: Immunohistochemistry showed that the expression of beta-arrestin2 increased along with the severity of liver fibrosis in liver fibrotic patients. We demonstrated that the expression of beta-arrestin2 was positively correlated with the collagen levels in porcine serum induced liver fibrosis. Loss of beta-arrestin2 results in inhibition of matrix deposition and protected liver function. In addition, our in vitro study showed that beta-arrestin2 overexpression significantly increased the production of ECM and activation of Smad2/3, Akt in activated HSCs. Conclusions: These data implicate beta-arrestin2 as mediators of development of hepatic fibrosis, thus selective targeting of beta-arrestin2 might present as a potential strategy for the treatment of hepatic fibrosis. 